\title{
Pacific
}

Journal of

Mathematics

\section{ON THE COMPACTNESS OF A CLASS OF RIEMANNIAN MANIFOLDS}

\author{
ZHIYONG GAO AND GUOJUN LIAO
}




\title{
ON THE COMPACTNESS OF A CLASS OF RIEMANNIAN MANIFOLDS
}

\author{
ZhiYong GaO and GuOJun Liao
}

A class of Riemannian manifolds is studied in this paper. The main conditions are 1) the injectivity is bounded away from $0 ; 2$ ) a norm of the Riemannian curvature is bounded; 3) volume is bounded above; 4) the Ricci curvature is bounded above by a constant divided by square of the distance from a point. Note the last condition is scaling invariant. It is shown that there exists a sequence of such manifolds whose metric converges to a continuous metric on a manifold.

Introduction. Let $\mathcal{L}=\mathcal{L}\left(H, K, V, n, i_{0}\right)$ be the set of $n$-dimensional Riemannian manifolds $(M, g)$, s.t.,

(0.1) $M$ is diffeomorphic to $\left(B_{2}, g_{0}\right)$, the standard Euclidean ball of radius 2 , center $=0$;

(0.2) $(M, g)$ has $C^{\infty}$ curvature tensor in $M$;

(0.3) for any $x \in M$, the Ricci curvature at $x|\operatorname{Ric}(g)(x)| \leq H r^{-2}$, where $r=\operatorname{dist}(x, 0)$;

(0.4) the injectivity of $(M, g) \geq i_{0}>0$;

(0.5) $\int_{M}|R m(g)|^{\frac{n}{2}} d g<K$;

(0.6) volume of $(M, g) \leq V$.

In the case when the condition (0.3) is replaced by $|R i c(g)| \leq H$, and (0.6) is replaced by a diameter bound, a compactness property is proved by the first author in a more general setting. The purpose of this paper is to extend some of his results to the present situation where the bound om Ricci curvature of $(M, g)$ blows up like $r^{-2}$ at a point. As an application, we will discuss the compactness of orbifolds with a finite number of singularities. 
The main result is:

TheOREM 0.7. Let $\left(M_{k}, g_{k}\right) \in \mathcal{L}, k=1,2,3, \ldots$ Then there exists a subsequence (again denoted by $\left(M_{k}, g_{k}\right)$ ), a $C^{\infty}$ manifold $M^{\prime}$ diffeomorphic to $B_{2}(0)$, and a $C^{0}$ metric $g^{\prime}$ on $M^{\prime}$ s.t. $g_{k} \rightarrow g^{\prime}$ in $C^{0}$-norm on $M^{\prime}$ and the convergence is in $C^{1, \alpha}$-norm away from 0 .

In Section 1 we study the geodesic balls centered at 0 . A compactness estimate of the metric $g$ will be derived. In Section 2, a small geodesic sphere is shown to have a small diameter. In Section 3 , some $L^{n / 2}$-curvature pinching results are derived, which will be used in Section 4 to show the existence of harmonic coordinates. We will prove in Section 4 the above main result and a slightly different version.

In the definition of $\mathcal{L}$, if $(0.3)$ is replaced by a 1 -sided condition

$$
\operatorname{Ric}(g) \geq-H r^{-2} g
$$

then the above compactness result should be modified as follows. Denote the set of such Riemannian manifolds by $\mathcal{L}^{\prime}$.

TheOREM 0.8. Let $\left(M_{k}, g_{k}\right) \in \mathcal{L}^{\prime}, k=1,2,3, \ldots$ Then there exists a subsequence of $\left(M_{k}, g_{k}\right)$, which converges in $C^{\circ}$-norm to a $C^{\infty}$ manifold $M^{\prime}$ with a $C^{\circ}$ metric $g^{\prime}$.

1. In this section, we assume that for some $H>0, \quad i_{0}>0$, $(M, g)$ is a Riemannian manifold diffeomorphic to $B_{2}$ satisfying

$$
\begin{gathered}
R i c(g) \geq-H r^{-2} g \\
i n j(g) \geq i_{0}>0 .
\end{gathered}
$$

Let $B_{\rho}(0)=\{x \in M \mid d(0, x) \leq\}$ be the geodesic ball of $M$ centered at 0 . Consider a geodesic polar coordinate system $\left\{r, x^{1}, \cdots, x^{n-1}\right\}$ on $B_{\rho}(0)$, we have

$$
d s(g)^{2}=d r^{2}+\sum_{i=1}^{n-1} g_{i j}(r, x) d x^{i} d x^{j}
$$




$$
R_{i r r j}=-\frac{1}{2} \frac{\partial^{2}}{\partial r^{2}} g_{i j}(r, x)+\frac{1}{4} \sum g^{k l} \frac{\partial}{\partial r} g_{i k} \frac{\partial}{\partial r} g_{\jmath l}
$$

For the Ricci curvature in the radial direction, we have

$$
R_{r r}=-\frac{\partial^{2}}{\partial r^{2}} \ln \sqrt{g(r)}-\frac{1}{4}\left|\frac{\partial}{\partial r} g(r)\right|_{g(r)}^{2}
$$

where $g(r)=g(r, x)$,

$$
\sqrt{g} d V_{0}=\sqrt{\operatorname{det}\left(g_{(} i j\right)} d x^{1} \wedge \ldots \wedge d x^{n-1},
$$

( $d V_{0}=$ the volume element of the standard Euclidean sphere) and

$$
\left|\frac{\partial g}{\partial r}\right|_{g}^{2}=\sum g^{i j} g^{k l} \frac{\partial}{\partial r} g_{i j} \frac{\partial}{\partial r} g_{k l} .
$$

We start out with the following estimate:

Proposition 1.7. For $\rho \leq \frac{i_{0}}{2}$, there exists $C_{1}=C_{1}(H, n)>0$ s.t. $\int_{0}^{\rho} r^{2}\left|\frac{\partial}{\partial r} g\right|^{2} d r \leq C_{1} \rho$.

Proof. The function is essentially the same as that given in [12], p.5-6. For any piecewise $C^{\infty}$ function $\phi$ of $r$ with $\phi(\rho)=0$, we have

$$
\begin{aligned}
& \left(\frac{1}{4}-\epsilon\right) \int_{0}^{\phi} r^{2} \phi^{2}\left|\frac{\partial}{\partial r} g\right|^{2} d r \\
& \leq \frac{n-1}{2 \epsilon} \int_{0}^{\rho}\left(r^{2} \phi^{\prime 2}+\phi^{2}\right) d r-\int_{0}^{\rho} r^{2} \phi^{2} R_{n} d r
\end{aligned}
$$

Take $\epsilon=\frac{1}{8}, \phi=\rho-r$, and use $-R_{r r} \leq H r^{-2}$, we get

$$
\begin{aligned}
& \int_{0}^{\phi} r^{2}(\phi-r)^{2}\left|\frac{\partial}{\partial r} g\right|^{2} d r \\
& \leq 32(n-1) \int_{0}^{\phi}\left(r^{2}+(\phi-r)^{2}\right) d r+H \int_{0}^{\phi}\left(r^{2}(\phi-r)^{2}\right) r^{-2} d r \\
& \leq C(H, n) \rho^{3} .
\end{aligned}
$$


Thus,

$$
\int_{0}^{\frac{\phi}{2}} r^{2}\left|\frac{\partial}{\partial r} g\right|^{2} d r \leq \frac{1}{\left(\frac{\rho}{2}\right)^{2}} \int_{0}^{\phi} r^{2}(\phi-r)^{2}\left|\frac{\partial}{\partial r} g\right|^{2} d r \leq \frac{1}{2} C_{1}(H, n) \rho .
$$

Proposition 1.9. There exists $C_{2}=C_{2}\left(H, i_{0}, n\right)>0$ s.t. for any $r \in\left(0, \frac{i_{0}}{2}\right)$, we have

$$
r\left|\frac{\partial}{\partial r} \ln \sqrt{g}\right| \leq C_{2}
$$

Proof. From (1.5) and integration by parts,

$$
\int_{0}^{\phi} r^{2} R_{n} d r=-\frac{1}{2} r^{2} \frac{\partial}{\partial r} \ln g+\frac{1}{2} \int_{0}^{\phi} 2 r \frac{\partial}{\partial r} \ln g-\frac{1}{2} \int_{0}^{\phi} r^{2}\left|\frac{\partial}{\partial r} g\right|^{2} d r .
$$

Thus

$$
\begin{aligned}
\frac{1}{2} r^{2} \frac{\partial}{\partial r} & \ln \sqrt{g} \leq H \int_{0}^{\phi} r^{-2} r^{2} d r+\frac{1}{4} C_{1} r+\left(\int_{0}^{\phi} r^{2}\left|\frac{\partial}{\partial r} \ln g\right|^{2}\right)^{\frac{1}{2}} r^{\frac{1}{2}} \\
& \leq \frac{1}{3} H r+\frac{1}{4} C_{1} r+(n-1)^{\frac{1}{2}}\left(\int_{0}^{\phi} r^{2}\left|\frac{\partial}{\partial r} g\right|^{2} d r\right)^{\frac{1}{2}} r^{\frac{1}{2}} \\
& \leq C_{2}\left(H, i_{0}, n\right) r .
\end{aligned}
$$

Next we study the induced metric $g(r)=\sum g_{i j}(r, x) d x^{i} d x^{j}$ on the geodesic sphere

$$
S_{r}(0)=\{x \in M: d(x, 0)=r\}, \quad r \leq \frac{i_{0}}{2} .
$$

Proposition 1.10. There exists $C_{3}=C_{3}(H, n)>0$ s.t. for $0<r_{1}<r_{2} \leq \frac{i_{0}}{2}$, we have

$$
e^{C_{3} r_{2} r_{1}^{-1}} g\left(r_{1}\right) \leq g\left(r_{2}\right) \leq e^{C_{3} r_{2} r_{1}^{-1}} g\left(r_{1}\right) .
$$


Proof. From Proposition 1.7, we have, for any vector $\nu=\left(\nu^{1}, \ldots, \nu^{n}\right) \in T S_{1}$,

$$
\begin{aligned}
\left|\ln \frac{h\left(r_{2}\right)}{h\left(r_{1}\right)}\right| & \leq \int_{r_{1}}^{r_{2}}\left|\frac{\partial}{\partial r} \ln h(r)\right| d r \leq\left(\int_{r_{1}}^{r_{2}}\left|\frac{\partial}{\partial r} g\right| r d r\right) r_{1}^{-1} \\
& \leq \sqrt{r_{2}}\left(C_{1} r_{2}\right)^{\frac{1}{2}} r_{1}^{-1}=\sqrt{C_{1}} \frac{r_{2}}{r_{1}}
\end{aligned}
$$

where $h(r)=g_{i j}(r) d \nu^{i} d \nu^{j}$. Hence $e^{C_{3} r_{2} r_{1}^{-1}} \leq \frac{h\left(r_{2}\right)}{h\left(r_{1}\right)} \leq e^{C_{3} r_{2} r_{1}^{-1}}$, where $c_{3}=\sqrt{c_{1}}$.

Before we go any further, let us make some remarks regarding conditions (0.3) and (0.5). Let $\tau>0$ be small. Define a new metric $g^{\tau}$ on $M$ by $g^{\tau}(x)=\tau^{-2} g(\tau x)$.

REMARK.

If $g$ satisfyes $(0.3)^{\prime}$, so does $g^{\tau}$.

$$
\int_{B_{1}}\left|R\left(g^{\tau}\right)\right|^{\frac{n}{2}} d g^{\tau}=\int_{B_{\tau}}|R(g)|^{\frac{n}{2}} d g
$$

Therefore, by a scaling of this type if necessary, we can assume that $g$ satisfies $(0.3)$ and $(0.5)$ with $K \ll 1$.

Once we have Proposition 1.10 we can control the $L^{n / 2}$ norm of the Riemannian curvature tensor $R m(r)$ of $g(r)$, the induced metric on $S(0, r)$.

TheOrem 1.13. If $(M, g) \in \mathcal{L}^{\prime}$ then for any $\rho \leq \frac{i_{0}}{4}$, there exist $r_{\rho} \in\left(\frac{\rho}{2}, \rho\right), \quad C_{4}=C_{4}\left(H, K, i_{0}, n\right)>0$, s.t.

$$
\int_{S\left(0, r_{\rho}\right)}\left|R m\left(r_{\rho}\right)\right|_{g\left(r_{\rho}\right)}^{\frac{n}{2}} d g\left(r_{\rho}\right) \leq C_{4} r_{\rho}^{-1}
$$

Proof. By Lemma 1.17 in $[\mathbf{1 2}], \exists C_{5}=C_{5}\left(H, i_{0}, n\right)$ s.t. for $\rho<\frac{i_{0}}{4}$,

$$
\int_{\frac{\rho}{2}}^{\rho}\left|\frac{\partial}{\partial r} g\right|^{n} d r \leq C_{5}\left(\frac{1}{\rho^{n}}+\int_{\frac{\rho}{2}}^{\rho}|R m(g)|^{\frac{n}{2}} d r\right) .
$$


From Proposition 1.10, there exists $C=C\left(H, i_{0}, n\right)$ s.t.

$$
C^{-1} \sqrt{g}(\rho) \leq \sqrt{g}(r) \leq C_{3} \sqrt{g}(\rho)
$$

for $r \in\left(\frac{\rho}{2}, \rho\right)$, i.e., $\sqrt{g}(r)$ is equivalent to $\sqrt{g}(\rho)$. Thus for some constant $C_{6}=C_{6}\left(H, i_{0}, n\right)>0$, we have

$$
\int_{\frac{\rho}{2}}^{\rho}\left|\frac{\partial}{\partial r} g\right|^{n} \sqrt{g}(r) d r \leq C_{6}\left(\rho^{-n} \sqrt{g}(\rho)+\int_{\frac{\rho}{2}}^{\rho}|R m(g)|^{\frac{n}{2}} \sqrt{g}(r) d r\right) .
$$

Integrating over $S_{\rho}(0)$, we get

$$
\int_{B_{\rho} \backslash B_{\frac{\rho}{2}}}\left|\frac{\partial}{\partial r} g\right|^{n} d g \leq C_{6} \rho^{-n} \int_{S_{\rho}} d g(\rho)+C_{6} \int_{B_{\rho}}|R m(g)|^{\frac{n}{2}} d g .
$$

Taking $\rho=\frac{i_{0}}{4}$, we get

$$
\int_{B_{\frac{z_{0}}{4}} \backslash B_{\frac{z_{0}}{8}}}\left|\frac{\partial}{\partial r} g\right|^{n} d g \leq C_{6}\left(\frac{i_{0}}{4}\right)^{-n} \operatorname{vol}\left(S_{\frac{z_{0}}{4}}\right)+C_{6} \int_{B_{\frac{z_{0}}{4}}}|R m(g)|^{\frac{n}{2}} d g .
$$

By Bishop's volume estimate [1], $\exists C_{7}=C_{7}\left(H, i_{0}, n\right)$ s.t. $\left.\operatorname{vol}\left(S_{\frac{i_{0}}{4}}\right)\right) \leq C_{7}$. Thus we get a constant $C_{8}=C_{8}\left(H, i_{0}, n\right)>0$ s.t.

$$
\int_{B_{\frac{z_{0}}{4}} \backslash B_{\frac{z_{0}}{8}}}\left|\frac{\partial}{\partial r} g\right|^{n} d g \leq C_{8}+C_{8} \int_{B_{\frac{i_{0}}{4}}}|R m(g)|^{\frac{n}{2}} d g .
$$

Define $g^{\tau}=r^{-2} g$ with $r=\frac{4 \rho}{i_{0}}$. Noticing that $\operatorname{Ric}\left(g^{\tau}\right) \geq-H r^{-2}$, $\operatorname{inj}\left(g^{\tau}\right) \geq i_{0}$, we can apply (1.16) to $g^{\tau}$. By the scaling invariance of $(1.16)$, we get

$$
\begin{aligned}
\int_{B_{\rho} \backslash B_{\frac{\rho}{2}}}\left|\frac{\partial}{\partial r} g\right|^{n} d g & =\int_{B_{\frac{z_{0}}{4}} \backslash B_{\frac{i_{0}}{8}}}\left|\frac{\partial}{\partial r} g^{\tau}\right|^{n} d g^{\tau} \\
& \leq C_{8}+C_{8} \int_{B_{\frac{z_{0}}{4}}}\left|R m\left(g^{\tau}\right)\right|^{\frac{n}{2}} d g^{\tau} \\
& =C_{8}+C_{8} \int_{B_{\rho}}\left|R m\left(g^{\tau}\right)\right|^{\frac{n}{2}} d g \\
& \leq C_{8}+C_{8} K=C_{9} .
\end{aligned}
$$


Hence

$$
\int_{\frac{\rho}{2}}^{\rho}\left(\int_{S_{r}}\left|\frac{\partial}{\partial r} g\right|^{n} d g(r)\right) d r \leq C_{9}
$$

(1.17) and the Gauss formula on $S$,

$$
R m(g)_{i j k l}=R m(g(r))_{i j k l}+\frac{1}{4}\left(\frac{\partial}{\partial r} g_{i k} \frac{\partial}{\partial r} g_{j l}-\frac{\partial}{\partial r} g_{j k} \frac{\partial}{\partial r} g_{i l}\right)
$$

imply that there exists a constant $C=C\left(H, K, i_{0}, n\right)>0$ s.t.

$$
\begin{aligned}
& \int_{\frac{\rho}{2}}^{\rho}\left(\int_{S_{r}}|R m(g(r))|^{\frac{n}{2}} d g(r)\right) d r \\
& \quad \leq C+C \int_{\frac{\rho}{2}}^{\rho}\left(\int_{S_{r}}|R m(g)|^{\frac{n}{2}} d g(r)\right) d r \\
& \quad \leq C+C K .
\end{aligned}
$$

This implies the existence of $r_{\rho} \in\left[\frac{\rho}{2}, \rho\right]$ and $C_{4}=C_{4}\left(H, K, i_{0}, n\right)>$ 0 s.t.

$$
\int_{S_{r_{\rho}}}\left|R m\left(r_{\rho}\right)\right|^{\frac{n}{2}} d g\left(r_{\rho}\right) \leq C_{4} r_{\rho}^{-1}
$$

We now state and prove the compactness estimate of the induced metric on small geodesic spheres.

Let $(M, g) \in \mathcal{L}^{\prime}, \quad \rho \leq \frac{i_{0}}{4}, \quad$ let $\quad r_{\rho} \in\left[\frac{\rho}{2}, \rho\right] \quad$ as in Theorem 1.13. We have the following

THEOREM 1.18. There exists $C_{10}=c_{10}\left(H, K, i_{0}, n\right)>0$ and a $C^{\infty}$ Riemannian metric $h\left(r_{\rho}\right)$ on the geodesic sphere $S_{r_{\rho}}$ s.t.

$$
\begin{gathered}
C_{10}^{-1} g\left(r_{\rho}\right) \leq r_{\rho}^{2} h\left(r_{\rho}\right) \leq C_{10} g\left(r_{\rho}\right) \\
\left|R m\left(h\left(r_{\rho}\right)\right)\right| \leq C_{10} .
\end{gathered}
$$

Proof. Proposition 1.10 and Theorem 1.13 are sufficient for carrying through the argument in [12]. 
2. In this section, we show that the diameter of a small geodesic sphere is small. More precisely,

TheOREM 2.1. There exists $C_{11}=C_{11}\left(H, K, i_{0}, V, n\right)$ s.t. for any $(M, g) \in \mathcal{L}^{\prime}, \quad$ any $\quad r \in\left(0, \frac{i_{0}}{2}\right), \quad \operatorname{diam}(g(r)) \leq C_{11} r$.

Proof. First observe that there exists a constant $C=C\left(H, K, i_{0}, V, n\right)>0$ s.t.

$$
\operatorname{diam}\left(S_{\frac{i_{0}}{4}}\right) \leq C
$$

To prove (2.2), we normalize by scaling so that $i_{0}=4$. Let $\gamma$ be a minimal geodesic on the geodesic sphere $S_{1}(0)$. We show that there exists $\tilde{C}=\tilde{C}\left(H, i_{0}, V\right)$ s.t.

$$
\text { length } \gamma \leq \tilde{C}
$$

Let $\alpha$ be any curve in the annulus $B_{\frac{3}{2}}(0) \backslash B_{\frac{1}{2}}(0)$ s.t. for $0 \leq$ $t_{1}<t_{2}<\cdots \leq 1, \quad \alpha \mid\left[t_{i}, t_{i+1}\right]$ is a minimal geodesic in the annulus. The geodesic balls centered at $\gamma\left(t_{i}\right)$ with radius $\delta$ can be made mutually disjoint by choosing $\delta>0$ sufficiently small. Let $N$ be the number of these balls. By Gromov's relative volume estimate [6], the volume of each small bal is bounded from below by a constant $C^{\prime}=C^{\prime}\left(H, i_{0}, V, n\right)$. But the total volume of the mannifold $M$ is bounded from above by $V$ (cf. (0.6)). Hence $N \leq V / C^{\prime}$. Since the induced metric $g\left(r_{1}\right)$ and $g\left(r_{2}\right)$ are equivalent (by Proposition 1.10), we can project $\alpha \mid\left[t_{i}, t_{i+1}\right]$ into $S_{1}(0)$, to get $(2.2)$.

Next, apply (2.2) to the metric $g^{\tau}$ defined by $g^{\tau}(x)=\tau^{-2} g(\tau x)$. By scaling properties, we get

$$
\operatorname{diam}(g(r)) \leq C \frac{4 r}{i_{0}}
$$


3. Let $(M, g)$ be in $\mathcal{L}^{\prime}$. As before we use the geodesic polar coordinates at 0 , i.e.,

$$
g=d r^{2}+\sum_{i, j=1}^{n-1} g_{i j}(x, r) d x^{i} d x^{j}=d r^{2}+g(r),
$$

where $g(r)=g(x, r)$ is the induced metric on the geodesic sphere $S_{r}(0)$.

We will begin with the following estimate:

Proposition 3.1. For $\rho \leq \frac{i_{0}}{4}, \quad \eta \in(0, \rho)$, we have

$$
\begin{gathered}
\int_{T\left(\frac{\eta}{4}, \frac{\eta}{2}\right)}\left(\max _{\eta \leq \rho} \int_{S(x, r)}\left|B(x, r)+\frac{1}{r} g(x, r)\right|^{\frac{n}{2}} d g(r)\right) d g(x) \\
\leq C(H, n, \eta, \rho) \int_{B(\rho+\eta)}\left|R_{m}(g)\right|^{\frac{n}{2}} d g,
\end{gathered}
$$

where $B(x, r)$ is the second fundamental form of $S(x, r)$,

$$
T\left(\frac{\eta}{4}, \frac{\eta}{2}\right)=\left\{x \in M \mid \operatorname{dist}(x, 0) \in\left(\frac{\eta}{4}, \frac{\eta}{2}\right)\right\} .
$$

Proof. Let $x \in T\left(\frac{\eta}{4}, \frac{\eta}{2}\right), \quad y \in M$ s.t. $d(x, y)=\rho \leq \frac{\imath_{0}}{2}$. Let $\gamma$ be the minimal geodesic from $x$ to $y$ with $\gamma(0)=x, \quad \gamma(\rho)=$ $y, \quad d(x, y)=\rho$. Observe that, as a consequence of Proposition 1.10, there exists a constant $C_{12}=C_{12}\left(H, i_{0}, n\right)>0$ s.t. for any Jacobi field $X$ on $\gamma$ with $X(\gamma(0))=0, \quad<X(\gamma(l)), \gamma^{\prime}(l)>=0$, we have

$$
|X(\gamma(t))| \leq C_{12}|X(\gamma(l))|
$$

$\forall t \in[0, l]$, where $l=$ the length of $\gamma$.

Let $E$ be the parallel vector field along $\gamma$ with

$$
E(\gamma(l))=X(\gamma(l))
$$

then the vector field $A$, defined by $A=X-\frac{t}{l} E$, is again a Jacobi field. Assume $|X(\gamma(l))|=1$. We have

$$
\begin{aligned}
\int_{0}^{l}\left|A^{\prime}\right|^{2} & =\int_{0}^{l}<A^{\prime \prime}, A>d t \leq \int_{0}^{l}|R m\|X\| A| d t \\
& \leq C_{12}\left(C_{12}+1\right) \int_{\gamma}|R m|=C_{13} \int_{\gamma}|R m|
\end{aligned}
$$


where $C_{13}=C_{13}\left(H, i_{0}, n\right)$.

Next, by a cut-off function argument, one can show that (c.f. [12], p.31)

$$
\left|A^{\prime}\right|^{2}(\gamma(l)) \leq C_{14} \int_{\gamma}|R m|^{2}
$$

We claim that there exists $C_{15}=C_{15}\left(H, K, i_{0}, n\right)$ s.t.

$$
\left|B(x, r)+\frac{1}{l} g(\gamma(l))\right|^{2}(\gamma(l)) \leq C_{15} \int_{\gamma}|R m|^{2} .
$$

To see this, let $X, Y$ be vector fields on $S(x, l)$ s.t.

$$
|X(\gamma(l))|=|Y(\gamma(l))|=1
$$

and let $E, \bar{E}$ be parallel vector fields on $\gamma$ with

$$
\begin{aligned}
& E(\gamma(l))=X(\gamma(l)), \\
& \bar{E}(\gamma(l))=Y(\gamma(l)) .
\end{aligned}
$$

Extended $X, Y$ to the geodesic ball $B(x, l)$ s.t. they are Jacobi fields on each radial geodesic. Then, clearly $B(X, Y)=$ $\left.-<\nabla_{\gamma^{\prime}}, X, Y\right\rangle=-\left\langle X^{\prime}, Y>\right.$. We have, from (3.2), that

$$
\begin{aligned}
& \left|B(X, Y)+\frac{1}{l}<X, Y>\right|^{2}(\gamma(l)) \\
& =\left|<X^{\prime}, Y>-\frac{1}{l}<E, Y>\right|^{2}(\gamma(l)) \\
& =\left|<X^{\prime}-\frac{1}{l} E, Y>\right|^{2}(\gamma(l)) \\
& \leq C_{14}|Y(\gamma(l))|^{2} \int_{\gamma}|R m|^{2}=C_{14} \int_{\gamma}|R m|^{2} .
\end{aligned}
$$

To finish the proof, we define $f(x, y)$, for $x, y$ with $d(x, y)=\rho+\frac{\eta}{2} \leq$ $\frac{i_{0}}{2}$, by

$$
f(x, y)=\max _{\eta \leq r \leq \rho}\left|B(x, r)+\frac{1}{r} g(x, r)\right|^{\frac{n}{2}}(\gamma(r)),
$$

where $\gamma$ is the minimal geodesic from $x$ to $y, \quad r=$ distance from $x$. 
Let

$$
\Omega=\bigcup_{x \in T\left(\frac{\eta}{4}, \frac{\eta}{2}\right)} S\left(x, \rho+\frac{\eta}{2}\right) \subset M
$$

and

$$
\Sigma=\bigcup_{x \in T\left(\frac{\eta}{4}, \frac{\eta}{2}\right)}\left(x, S\left(x, \rho+\frac{\eta}{2}\right)\right) \subset M \times M .
$$

Then

$$
\begin{aligned}
\int_{\Sigma} \int f(x, y) & =\int_{x \in T\left(\frac{\eta}{4}, \frac{\eta}{2}\right)}\left(\int_{S\left(x, \rho+\frac{\eta}{2}\right)} f(x, y) d g_{x}(y)\right) d g(x) \\
& =\int_{\Omega}\left(\int_{\Omega_{y}} f(x, y) d g_{y}(x)\right) d g(y)
\end{aligned}
$$

where $g_{x}$ is the induced metric of $S\left(x, \rho+\frac{\eta}{2}\right)$, and $\Omega_{y}=T\left(\frac{\eta}{4}, \frac{\eta}{2}\right) \cap$ $S\left(y, \rho+\frac{\eta}{2}\right) \subset S\left(y, \rho+\frac{\eta}{2}\right)$. We have

$$
\int_{\Sigma} \int f(x, y) \leq \int_{\Omega}\left(\int_{\Omega_{y}} f(x, y) d g_{y}(x)\right) d g(y) .
$$

Define $\bar{\gamma}(t)=\gamma(t)$ for $t \in[0, \rho]$. From (3.3) we get

$$
\begin{aligned}
& \int_{\Omega_{y}} f(x, y) d g_{y}(x) \\
& \leq C(H, \eta, \rho) \int_{\Omega_{y}}\left(\int_{\bar{\gamma}}|R m(g)|^{\frac{n}{2}}\right) d g_{y} \\
& \leq C(H, \eta, \rho) \int_{\delta}^{\rho+\delta}\left(\int_{\Omega_{y}}|R m(g)|^{\frac{n}{2}}\left(\gamma\left(\rho+\frac{\eta}{2}-t\right)\right) d g_{y}\right) d t .
\end{aligned}
$$

By Proposition 1.10,

$$
d g_{y}\left(\gamma\left(\rho+\frac{\eta}{2}-t\right)\right) \geq C\left(H, n, \frac{\rho}{\eta}\right) d g_{y}(x)
$$

Therefore

$$
\int_{\Omega_{y}} f(x, y) d g_{y}(x) \leq C\left(H, n, \eta, \frac{\rho}{\eta}\right) \int_{B(\rho+\eta)}|R m(g)|^{\frac{n}{2}} d g .
$$


Finally we have

$$
\begin{aligned}
\int_{\Omega_{y}} f(x, y) & \leq C\left(H, n, \eta, \frac{\rho}{\eta}\right) \operatorname{vol}\left(T\left(\frac{\eta}{4}, \rho+\eta\right)\right) \int_{B(\rho+\eta)}|R m(g)|^{\frac{n}{2}} d g \\
& \leq C\left(H, n, \eta, \frac{1}{\eta}, \rho, V, i_{0}\right) \int_{B(\rho+\eta)}|R m(g)|^{\frac{n}{2}} d g
\end{aligned}
$$

Let $\dot{R} m(r)$ be the scalar curvature free curvature tensor of $g(r)$. We have the following proposition.

Proposition 3.4. For any $x \in T\left(\frac{\eta}{4}, \frac{\eta}{2}\right)$, where $\eta \in(0, \rho)$ with $\rho \leq \frac{i_{0}}{4}$, we have

$$
\begin{aligned}
& \int_{\eta}^{\rho}\left(\int_{S(x, r)}|\dot{R} m(r)|^{\frac{n}{4}} d g_{x}(r)\right) d r \\
& \leq C\left(H, n, \eta, \rho, i_{0}\right)\left(\left(\int_{B_{x}(\rho)}|R m(g)|^{\frac{n}{2}} d g\right)^{\frac{1}{2}}\right. \\
& \quad+\left(\max _{\eta \leq \rho} \int_{S(x, r)}\left|A(r)+\frac{1}{r} g_{x}(r)\right|^{\frac{n}{2}} d g_{x}(r)\right)^{\frac{1}{2}} \\
& \left.\quad+\max _{\eta \leq \rho} \int_{S(x, r)}\left|A(r)+\frac{1}{r} g_{x}(r)\right|^{\frac{n}{2}} d g_{x}(r)\right)
\end{aligned}
$$

Proof. $\dot{R} m(r)$ can be expressed as

$$
\begin{aligned}
& (\dot{R} m(r))_{i j k l} \\
& =(R m(r))_{i j k l}-\frac{R(r)}{(n-1)(n-2)}\left(g_{i k}(r) g_{j l}(r)-g_{i l}(r) g_{j k}(r)\right),
\end{aligned}
$$

where $R(r)$ is the scalar curvature of $g(r)$. We have

$$
\int_{S(x, r)}\left|B_{i k}(r) B_{j l}(r)-\frac{1}{r^{2}} g_{i k}(r) g_{j l}(r)\right|^{\frac{n}{4}} d g(r)
$$




$$
\begin{aligned}
= & \int_{S(x, r)} B_{i k}(r)\left(B_{j l}(r)+\frac{1}{r} g_{\jmath l}(r)\right) \\
& -\frac{1}{r} g_{j l}(r)\left(B_{i k}(r)+\frac{1}{r} g_{i k}(r)\right)^{\frac{n}{4}} d g(r) \\
\leq & C \int_{S(x, r)}|B|^{\frac{n}{4}}\left|B(r)+\frac{1}{r} g(r)\right|^{\frac{n}{4}} d g(r) \\
& +C \int_{S(x, r)}\left|B(r)+\frac{1}{r} g(r)\right|^{\frac{n}{4}} d g(r) \\
\leq C & \left(\int_{S(x, r)}\left|B(r)+\frac{1}{r} g(r)\right|^{\frac{n}{2}} d g(r)\right)^{\frac{1}{2}} \\
& +C \int_{S(x, r)}\left|B(r)+\frac{1}{r} g(r)\right|^{\frac{n}{2}} d g(r) .
\end{aligned}
$$

This implies that

$$
\begin{gathered}
\int_{S(x, r)}\left|\left(B_{i k} B_{j l}-B_{i l} B_{j k}\right)-\frac{1}{r^{2}}\left(g_{i k} g_{j l}-g_{i l} g_{j k}\right)\right|^{\frac{n}{4}} d g(r) \\
\leq C\left(H, K, i_{0}, n\right)\left(\int_{S(x, r)}\left|B(r)+\frac{1}{r} g(r)\right|^{\frac{n}{2}} d g(r)\right)^{\frac{1}{2}} \\
+C\left(H, K, i_{0}, n\right) \int_{S(x, r)}\left|B(r)+\frac{1}{r} g(r)\right|^{\frac{n}{2}} d g(r) .
\end{gathered}
$$

By Gauss formula,

$$
(R m(g))_{i j k l}=(R m(g(r)))_{i j k l}+B_{i k}(r) B_{\jmath l}(r)-B_{i l}(r) B_{j k}(r) .
$$

Therefore

$$
\begin{aligned}
& \int_{\eta}^{\rho}\left(\int_{S(x, r)} \mid R_{i \jmath k l}(g(r))-\frac{1}{r^{2}}\left(g_{i k}(r) g_{j l}(r)\right.\right. \\
& \left.\left.\quad-g_{i l}(r) g_{j k}(r)\right)\left.\right|^{f r a c n 4} d g(r)\right) d r \\
& \leq C(H, n, \eta, \rho)\left(\int_{B(x, \rho)}|R m(g)|^{\frac{n}{2}} d g\right)^{\frac{1}{2}} \\
& \quad+C(H, n, \eta, \rho)\left(\max _{\eta \leq r \leq \rho} \int_{S(x, r)}\left|B(r)+\frac{1}{r} g(r)\right|^{\frac{n}{2}} d g(r)\right)^{\frac{1}{2}} \\
& \quad+C(H, n, \eta, \rho)\left(\max _{\eta \leq r \leq \rho} \int_{S(x, r)}\left|B(r)+\frac{1}{r} g(r)\right|^{\frac{n}{2}} d g(r)\right) .
\end{aligned}
$$


Observe that

$$
\begin{aligned}
\int_{T_{x}(\eta, \rho)} & \left|R(r)-\frac{(n-1)(n-2)}{r^{2}}\right|^{\frac{n}{4}} d g \\
\leq & C\left(H, K, i_{0}, n, \eta, \rho\right)\left(\int_{B(x, \rho)}|R m(g)|^{\frac{n}{2}} d g\right)^{\frac{1}{2}} .
\end{aligned}
$$

Hence (3.4) follows immediately.

Proposition 3.5. For $0<\eta<\rho \leq \frac{i_{0}}{4}$, let $\left(M_{k}, g_{k}\right) \in$ $\mathcal{L}^{\prime}, \quad x_{k} \in M_{k} \quad$ with $\quad \operatorname{dist}\left(x_{k}, 0\right) \in\left(\frac{\eta}{4}, \frac{\eta}{2}\right)$. Assume

$$
\eta_{k}=\max _{\eta \leq r \leq \rho} \int_{S(x, r)}\left|B\left(x_{k}, r\right)+\frac{1}{r} g_{k}(r)\right|^{\frac{n}{2}} d g_{k}(r) \rightarrow 0
$$

and

$$
\mu_{k}=\int_{B\left(x_{k}, \rho\right)}\left|R m\left(g_{k}\right)\right|^{\frac{n}{2}} d g_{k} \rightarrow 0 \quad \text { as } \quad k \rightarrow \infty .
$$

Then there exists a diffeomorphism $\phi_{k}: S(1) \rightarrow S\left(x_{k}, \rho\right) \quad$ for each $k=1,2,3, \cdots$, s.t.

$$
\int_{S(1)}\left|\phi_{k}^{*} g_{k}(r)-r^{2} d \theta^{2}\right|^{\frac{n}{2}} d \theta \rightarrow 0
$$

uniformly for $\eta \leq r \leq \rho$, where $S(1)$ is the Euclidean unit sphere, and

$$
\left|\phi_{k}^{*} g_{k}(\rho)-\rho^{2} d \theta^{2}\right|_{C^{0}} \rightarrow 0 \quad \text { as } \quad k \rightarrow \infty
$$

Proof. Proposition 1.10 and Theorem 1.13 enable us to carry out the arguments in [12] (cf. 5.18, 5.21, and 5.25).

4. In this section we prove the existence of a controllable harmonic coordinate system under the smallness condition of the $L^{n / 2}$ norm of curvature tensor.

Proposition 4.1. For any $\eta \in(0,1)$, there exists $\epsilon=\epsilon\left(H, n, i_{0}, \eta\right)>0$ s.t. if $(M, g) \in \mathcal{L}$ satisfies $\int_{M}|R m(g)|^{\frac{n}{2}} d g \leq$ 
$\epsilon$, then there exists a diffeomorphism

$$
F=\left(h^{1}, h^{2}, \cdots, h^{n}\right): \quad T\left(1+\frac{\eta}{2}, \frac{3 \eta}{2}\right) \rightarrow T\left(1+\frac{\eta}{2}, \frac{3 \eta}{2}\right) \subset \mathbb{R}^{n}
$$

having the following properties:

(a) $\Delta=0$;

(b) $F^{-1}\left(T\left(1+\frac{\eta}{4}, \frac{\eta}{4}+\eta\right)\right) \supset T(1-\eta, 2 \eta)$ and the image of $F \supset$ $T\left(1+\frac{\eta}{4}, \frac{5 \eta}{4}\right)$;

(c) $\left|h^{i j}-\delta^{i j}\right|_{C^{0}}<\frac{\eta^{2}}{100 n}$ on $T\left(1+\frac{\eta}{4}, \frac{5 \eta}{4}\right) ; \quad$ where $h^{i j}=<\nabla h^{i}, \nabla h^{j}>$;

(d) $\left|d h^{i j}\right|_{C^{0}} \leq C(H, n, \eta)$ for some $\alpha \in(0,1)$ on $T\left(1+\frac{\eta}{4}, \frac{5 \eta}{4}\right)$;

(e) $\left.|| F\right|^{2}-r^{2} \mid \leq \frac{\eta}{100 n}$, where $|F|^{2}=\sum_{i}\left(h^{i}\right)^{2}, \quad r=\operatorname{dist}(x, 0)$;

(f) $\left\|d^{2} h^{i j}\right\|_{L^{q}} \leq C(H, n, \eta) \quad$ on $\quad T\left(1+\frac{\eta}{4}, \frac{5 \eta}{4}\right) \quad$ for some $\quad q>$ $n$.

Proof. Suppose for $k=1,2, \cdots, \quad\left(M_{g}, g_{k}\right) \in \mathcal{L} \quad$ with $\int_{M_{k}}\left|R m\left(g_{k}\right)\right|^{\frac{n}{2}} \leq \frac{1}{k}$.

Proposition 3.1 implies that $\exists y_{k} \in T\left(\frac{\eta}{2}, \frac{\eta}{4}\right)$ s.t.

$$
\begin{aligned}
\eta_{k} & =\max _{\eta \leq r \leq 1} \int_{S_{k}\left(y_{k}, r\right)}\left|B_{k}\left(y_{k}, r\right)+\frac{1}{r} g_{k}\left(y_{k}, r\right)\right|^{\frac{n}{2}} d g_{k}\left(y_{k}, r\right) \\
& \leq C\left(H, n, i_{0}, \eta, \frac{1}{\eta}\right) \int_{B_{2}}\left|R m\left(g_{k}\right)\right|^{\frac{n}{2}} d g_{k} \\
& \leq C k^{-1} .
\end{aligned}
$$

Proposition 3.5 implies that there exists $\phi_{k}: S_{1} \rightarrow S_{k}\left(y_{k}\right) \approx S_{1}$ s.t.

$$
\int_{T(1, \eta)}\left|\phi_{k}^{*} g_{k}-g_{0}\right|^{\frac{n}{2}} d g_{0}<C k^{-1}
$$

where $\phi_{k}$ has been extended trivially to $T(1, \eta), \quad g_{0}$ is the flat metric on $B_{1}$. In the Euclidean coordinates $x=\left(x^{1}, \cdots, x^{n}\right), \quad g_{0}=\delta_{i j}$.

Next we solve the Dirichlet problem

$$
\begin{cases}\Delta F=0 & \text { in } T(1, \eta) \\ F=x & \text { on } \partial T(1, \eta) .\end{cases}
$$


By Proposition 1.10, we can show (as in [14])

$$
\int_{T(1, \eta)}|\nabla F-\nabla x|_{g}^{2} d g \leq \frac{1}{k} C\left(H, n, \frac{1}{\eta}, \eta, i_{0}\right) .
$$

By a standard argument involving DeGiorgi-Nash-Moser iteration, it follows that $F$ is the desired diffeomorphism.

THEOREM 4.2. For each $M_{k}, g_{k} \in \mathcal{L}$, there exists, for $l=1,2, \cdots$, open sets $F_{k}(l) \subset M_{k}$ s.t. $F_{k}(l+1) \supset F_{k}(l)$ and $F_{k}(l) \cup B\left(l^{-1}\right)=M_{k}$. There also exists a diffeomorphism $\phi_{k}(l)$ for each pair of $k$ and $l: \quad \phi_{k}(l): T\left(1, l^{-1}\right) \subset \mathbb{R}^{n} \rightarrow F_{k}(l)$ such that $\phi_{k}(l)^{*} g_{k}$ converges in $C^{1, \alpha}$ norm to some $C^{1, \alpha}$ metric $g_{l}^{\prime}$ on $T\left(1, l^{-1}\right) \subset \mathbb{R}^{n}$.

Proof. By rescaling, we can assume that $g_{k}$ satisfies

$$
\int_{M_{k}}\left|R m\left(g_{k}\right)\right|^{\frac{n}{2}} d g_{k} \leq \epsilon
$$

where $\epsilon>0$ is given by Proposition 4.1. Therefore we have harmonic coordinates

$$
h^{k}: T_{k}\left(1+\frac{\eta}{2}, \frac{3 \eta}{2}\right) \subset M_{k} \rightarrow D(\eta)=T\left(1+\frac{\eta}{2}, \frac{3 \eta}{2}\right) \subset \mathbb{R}^{n},
$$

satisfying (a)-(f) of 4.1. Taking $\eta=l^{-1}$, by the Hölder estimate (d), we have, for each $l=1,2, \cdots$, a subsequence of $\left(M_{k}, g_{k}\right)$, denoted by $g_{k}(l)$, s.t. $g_{k}(l)$ converges in the $C^{2}$-norm on $T_{k}\left(1+\frac{\eta}{2}, \frac{3 \eta}{2}\right) \subset M$ to a $C^{1, \alpha}$ metric $g_{l}^{\prime}$ on $D(l)$. We can then take

$$
F_{k}(l)=T_{k}\left(1+\frac{\eta}{2}, \frac{3 \eta}{2}\right), \quad \eta=\frac{1}{l} .
$$

By passing to a subsequence if necessary, we can make $F_{k}(l+1) \supset$ $F_{k}(l)$.

THEOREM 4.3. Let $g^{\prime}$ be a metric on $M^{\prime} \cong B_{1} \mid\{0\}$ defined by $g^{\prime}(x)=g_{l}^{\prime}(x)$ if $x \in F_{k}(l)$. Then $g^{\prime}$ can be extended as a $C^{0}$ metric on $B_{1}$.

Proof. Theorem 2.1 says that the diameter of a small geodesic sphere around 0 is small. Hence 0 is the only possible singularity. To 
show that 0 is a removable singular point, let, for fixed $N=1,2, \cdots$,

$$
C(\rho, N)=\left\{x \in M^{\prime} \mid \frac{\rho}{N}<d(x, 0)<2 \rho\right\} .
$$

By Theorem 4.2, a subsequence $\left(M_{k}, g_{k}\right)$ converges to $M^{\prime}$ away from 0 . Thus for each $\rho, \exists k=k(\rho), \exists$ a submanifold $C_{k}(\rho, N) \subset$ $\left(M_{k}, g_{k}\right), \exists y_{\rho} \in C_{k}(\rho, N)$ s.t. $y_{\rho} \rightarrow x_{\rho} \in C(\rho, N)$ (with $\operatorname{dist}\left(x_{\rho}, 0\right)=$ $\rho)$, and such that

$$
\left.\left|\int_{C_{k}(\rho, N)}\right| R m\left(g_{k}\right)\right|^{\frac{n}{2}} d g_{k}-\int_{C(\rho, N)}\left|R M\left(g^{\prime}\right)\right|^{\frac{n}{2}} d g^{\prime} \mid \leq \rho^{2},
$$

and

$$
\left\|\left(\frac{1}{\rho} C(\rho, N), x_{\rho}\right)-\left(\frac{1}{\rho} C_{k}(\rho, N), y_{k}\right)\right\|_{C^{1, \alpha}}<\rho .
$$

By (0.5),

$$
\int_{C(\rho, N)}\left|R M\left(g^{\prime}\right)\right|^{\frac{n}{2}} d g^{\prime} \rightarrow 0 \quad \text { as } \quad \rho \rightarrow 0 .
$$

Consequently,

$$
\int_{C_{k}(\rho, N)}\left|R M\left(g_{k}\right)\right|^{\frac{n}{2}} d g_{k} \rightarrow 0 \quad \text { as } \quad \rho \rightarrow 0 .
$$

Therefore, from the zero pinching theorem of [12], it follows that $\left(\frac{1}{\rho} C_{k}(\rho, N), y_{\rho}\right)$ converges to a flat manifold $D_{N}$ in $C^{1, \alpha}$-norm as $\rho \rightarrow 0$. Thus $\left(\frac{1}{\rho} C(\rho, N), x_{\rho}\right)$ converges to $\left(D_{N}, e_{N}\right)$ in $C^{1, \alpha}$-norm. The direct union of $\left(D_{N}, e_{N}\right)$ has to be $(U(0), e)$ where 0 is the isolated singular point, $e$ is a unit vector in $\mid B b b R^{n}$, and $U(0)$ is a simply connected flat manifold since $\frac{1}{\rho} C(\rho, N)$ is the $C^{1, \alpha}$ limit of simply connected manifolds $\frac{1}{\rho} C_{k}(\rho, N)$. Hence $U(0) \cong B(2)-\{0\}$. Letting $N \rightarrow \infty$ have that $\left(\frac{1}{\rho} C(\rho, 0), x_{\rho}\right)$ converges to $\{B(2)-$ $\{0\}, e\}$ in $C^{1, \alpha}$-norm. It follows that $g^{\prime}$ can extend to a $C^{0}$ metric on $M^{\prime}$, diffeomorphic to $B_{1} \subset \mathbb{R}^{n}$.

REMARK. In the case $\left(M_{k}, g_{k}\right) \in \mathcal{L}^{\prime}$, we use Proposition $3.5 \mathrm{di}$ rectly in place of Proposition 4.1 and Theorem 4.2. This, combined with Theorem 4.3, proves Theorem (0.8).

REMARK. Let $O$ be the set of compact orbifolds with finitely many singular points, satisfying $(0.3)-(0.6)$. Let $\Gamma$ be the group 
acting on these orbifolds. We can lift a neighbourhood of each singular point via $\Gamma$ to $B^{n}$. It then follows from Theorem (0.7) that $O$ has the same compactness property.

\section{REFERENCES}

[1] R.Bishop, R.Crittenden, Geometry of Manifolds, Acad.Press, New York, (1964).

[2] M.Berger, Les varietes riemanniennes (1/4)-pincees, Ann. Scuola. Norm. sup. Pisa Sci. Fis. Mat., (3) 14 (1960), 160-170.

[3] C.B.Croke, Some isoperimetric inequalities and eigenvalue estimates, Ann. Sci. École Norm. Super., Paris, 13 (1980), 419-435.

[4] J.Cheeger, D.G.Ebin, Comparison Theorems in Riemannian Geometry, North-Holland Mathematical Library, 9, North-Holland, Amsterdam, (1975).

[5] J.Cheeger, D.Gromoll, On the structure of complete manifolds of nonnegative curvature, Ann. of Math., 96, No.3 (1972), 413-443.

[6] J.Cheeger, M.Gromov, M.Taylor, Finite propagation speed, kernel estimates for functions of the Laplace operator, and the geometry of complete Riemannian manifolds, J.Differential Geom., 17 (1982), 15-54.

[7] S.Y.Cheng, P.Li, S.T.Yau, On the upper estimate of the heat kernel of a complete Riemannian manifold, Amer. J. Math., 103 (1981), 1021-1063.

[8] H.Federer, Geometric Measure Theory, Springer-Verlag, Berlin (1969).

[9] M.Freedman, The topology of four-dimensional manifolds, J. Differential Geom., 17 (1982), 357-454.

[10] D.Gromoll, Differenzierbare Strukturen and Metriken positiver Krummung auf Spharen, Math. Ann., 164 (1966), 353-371.

[11] D.Gromoll, W.Klingenberg, W.Meyer, Riemannsahe Geometrie im Grossen, Second edition. Lect. Notes in Math., Springer-Verlag (1975).

[12] L.Z.Gao, $L^{n / 2}$ curvature pinching, J.Differential Geom., 32, No.3 (1990), 713-774.

[13] L.Z.Gao, Einstein metrics, J.Differential Geom., 32, No.1 (1990), 155184.

[14] L.Z.Gao, Convergence of Riemannian manifolds, Ricci pinching and $L^{n / 2}$ curvature pinching, J.Differential Geom., 32, No.2 (1990), 349-382.

[15] M.Gromov, J.Lafontaine, P.Pansu, Struture metrique pour les varietes Riemanniennes, Cedic/Fernand, Nathan (1981).

[16] M.Gromov, Manifolds of negative curvature, J.Differential Geom., 13 (1978), 223-230.

[17] M.Gromov, Groups of Polynomial Growth and Expanding Maps, Inst. Hautes Études Sci., (1986). 
[18] K.Grove, P.Petersen, Bounding homotopy types by geometry, Ann. of Math., 128 (1988), 195-206.

[19] M.Gromov, W.Thurston, Pinching constants for hyperbolic manifolds, Invent. Math., 89 (1987), 1-12.

[20] R.Greene, H.Wu, Lipschitz convergence of Riemannian manifolds, Pacific J.Math., 131 (1988), 119-141.

[21] G.Huisken, Ricci deformation of the metric on a Riemannian manifold, J.Differential Geom., 21 (1985),47-62.

[22] R.Hamilton, Three manifolds with positive Ricci curvature, J.Differential Geom., 17 (1982), 255-306.

[23] M.A.Kervaire, J.W.Milnor, Groups of homotopy sphere, I, Ann.of Math., (2) 77 (1963), 504-537.

[24] S.Kobayashi, K.Nomizu, Foundations of Differential Geometry, John Wiley, New York (1963).

[25] K.Klingenberg, Uber Riemannsche Mannigfaltigkeiten mit positiver Krummung, Comment. Math. Helv., 35 (1961), 47-54.

[26] P.Li, On Sobolev constant and the p-spectrum of a compact Riemannian manifold, Ann. Sci. École. Norm. Super., (4) 13 (1980),451-469.

[27] Min-Oo, Almost Einstein manifolds of negative Ricci curvature, J. Differential Geom., 32, No.2 (1990), $457-472$.

[28] S.Peters, Convergence of Riemannian manifolds, Compositio Math., 62 (1987), 3-16.

[29] E.A.Ruh, Curvature and differentiable structures on spheres, Comment. Math. Helv., 46 (1971), 127-136.

[30] E.A.Ruh, Riemannian manifolds with bounded curvature ratios, J. Differential Geom., 17 (1982), 643-653.

[31] H.E.Rauch, A contribution to differential geometry in the large, Ann. of Math., (2) 54 (1951), 38-55.

[32] L.Sibner, The isolated point singularity problem for the coupled YangMills equations in higher dimensions, Math. Ann., 271 (1985), 125-131.

[33] S.Smale, Generalized Poincare conjecture in dimensions greater than four, Ann. of Math., 74 (1961), 391-406.

[34] J.Wolf, Spaces of Constant Curvature, McGraw-Hill, New York (1967).

[35] Deane Yang, $L^{p}$ pinching and compactness theorems for compact Riemannian manifolds, preprint. 
Received December 5, 1990, revised April 8, 1991 and eccepted for publication November 18, 1991.

RICE UNIVERSITY

Houston, TX 77251

E-mail address: liao@utamat.uta.edu

AND

UNIVERSITY OF TEXAS

ARLINGTON, TX 76019-0408 



\section{PACIFIC JOURNAL OF MATHEMATICS}

Volume $166 \quad$ No. $1 \quad$ November 1994

Eigenvalue bounds and girths of graphs of finite, upper half-planes

NANCY JEANNE CELNIKER

On the compactness of a class of Riemannian manifolds

ZHIYONG GAO and GUOJUN LiAO

The distribution mod $n$ of fractions with bounded partial quotients

Douglas Austin Hensley

Paired calibrations applied to soap films, immiscible fluids, and

surfaces or networks minimizing other norms

GARY REID LAWLOR and FRANK MORGAN

Conformal repellors with dimension one are Jordan curves

R. DANIEL MAULDIN and MARIUSZ URBANSKI

Order of the identity of the stable summands of $\Omega^{2 k} S^{2 n+1}$

PAUl Silberbush

On a construction of pseudo-Anosov diffeomorphisms by sequences of 123 train tracks

ITARU TAKARAJIMA

On systems of generators of arithmetic subgroups of higher rank groups

T. N. VENKATARAMANA 